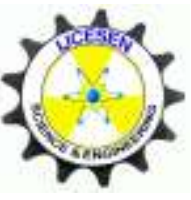

Copyright (C) IJCESEN
International Journal of Computational and

Experimental Science and $\boldsymbol{E N g i n e e r i n g}$

(IJCESEN)

Vol. 7-No.2 (2021) pp. 76-83

http://dergipark.org.tr/en/pub/ijcesen

Research Article

\title{
Changes in Interaction Between Accessory Protein 8 and IL17RA in UK Isolates Caused by Mutations in the SARS-CoV-2 Open Reading Frame 8
}

\author{
Ekrem AKBULUT* \\ Malatya Turgut Özal University, Engineering and Natural Sciences Faculty, Bioengineering Department, 44200, \\ Malatya-Turkey \\ * Corresponding Author : ekremakbulut@gmail.com ORCID: 0000-0002-7526-9835
}

\section{Article Info:}

DOI: $10.22399 /$ ijcesen.935624

Received : 10 May 2021

Accepted : 21 June 2021

$\underline{\text { Keywords }}$

SARS-CoV-2

COVID-19

Open Reading Frame 8

Accessory Protein 8

Mutation

ILI7RA

\begin{abstract}
:
SARS-CoV-2 is the infectous agent of COVID-19, one of the most important health problems of the twenty-first century. IL17RA is an crucial receptor in the generation of the host immune response. ORF8 is the viral accessory protein of SARS-CoV-2 that suppresses the host immune response. Mutations can alter the viral properties and clinical course of SARS-CoV-2. In this study, we investigated the changes that SARS-CoV-2 ORF8 mutations may cause in the interaction of IL17RA with ORF8. The study was carried out using 825 complete genome sequences from UK isolates. Mutation analyzes were performed using RDP4 and MEGAX. The protein model was created using the Swiss Model. Protein protein interaction was analyzed by Haddock ver 2.4. Analysis of changes in protein stability was performed using SDM2, mCSM stability and DUET tools. The change in ORF8 - ILI7RA binding affinity before and after the mutation was evaluated using mCSM-PPI2. We detected P30S, R52I, Y73C and L118V mutations in SARS-CoV-2 ORF8. Mutations have been shown to reduce protein stability and affinity. After the mutation, the binding dynamics of ORF8 to IL17RA were changed. Molecular attachment scores were $-78.0 \pm 3.4 \mathrm{kcal}^{\mathrm{mol}}{ }^{-1}$ and $-76.3 \pm 11.9 \mathrm{kcal}^{\mathrm{mol}} \mathrm{m}^{-1}$, for wild type and mutant, respectively. After the mutations, the hydrogen bond number and position between ORF8 and IL17RA changed. While establishing ten hydrogen bonds between the wild type and IL17RA, four hydrogen bonds were established between the mutant ORF8 and IL17RA. The decreased affinity between ORF8 and ILI7RA can be seen as a stronger immune response and a milder clinical course. Although our results contain important data for understanding ORF8, which is an important drug target, it needs to be repeated with in-vivo and crystallgraphy studies.
\end{abstract}

\section{Introduction}

Severe acute respiratory syndrome coronavirus 2 (SARS-CoV-2) has killed 3.27 million people since December 2019. SARS-CoV-2, which revealed the third largest coronavirus epidemic in the last 20 years, infected 160 million people [1,2]. The molecular basis of the severity and rapid spread of COVID-19 disease caused by SARS-CoV-2 is largely unknown [3]. Although prophylactic vaccine development studies have recently been successful in COVID-19 disease, the high mutation capacity and progressive evolutionary change of the virus genome pose the risk of overriding existing treatments [4]. SARS-CoV-2, which is of zoonotic origin such as severe acute respiratory syndrome coronavirus (SARS) and Middle East respiratory syndrome (MERS), may be a sign that members of this family may cause serious health problems in the future, and even epidemics with transition between species [5,6].

The SARS-CoV-2 genome consists of 16 nonstructural proteins, 4 structural proteins $(\mathrm{S}, \mathrm{M}, \mathrm{E}, \mathrm{N})$ and 5 accessory proteins [7]. Although the SARSCoV-2 genome is known as the genetic code, the functional role of many accessory proteins is not clearly $[8,9]$. Open Reading Frame-8 (ORF8) from SARS-CoV-2 is an accessory protein consisting of 366 nucleotides. Its evolutionary distance from other beta coronaviruses makes SARS-CoV-2 ORF8 an 
important research target [8]. ORF8 from SARS CoV-2 is a rapidly evolving accessory protein that has been proposed to interfere with immune responses [3]. ORF8 from SARS-CoV-2 disrupts antigen presentation and reduces the recognition and elimination of virus-infected cells [10]. ORF8 suppresses the immune response by reducing the expression of MHC I (HLA-A2) molecules. The major pathway for ORF8-mediated MHC-I downregulation is the lysosomal degradation. The proteins that ORF8 from SARS-CoV-2 interacts with in the host are localized in the endoplasmic reticulum [11]. This may result in the rearrangement of the ER traffic of host-ORF8 interactions during infection.

ORF8 from SARS-CoV-2 was shown to inhibit type I interferon $(I F N-\beta)$ activation and NF-kappa-B pathway [12]. Cytokine binding triggers the homotypic interaction of the ILI7RA and IL-17RC chains with the TRAF3IP2 adapter, leading to TRAF6-mediated activation of the NF-kappa-B and MAP kinase pathways, resulting in the activation of cytokines, chemokines, antimicrobial peptides and matrix metallocationproteinases that will ultimately generate strong immune inflammation [13-15].

ILI7RA involved in antiviral host defense through various mechanisms. IL17RA plays a role in the maintenance of the integrity of epithelial barriers during pathogen infection. It stimulates the production of antimicrobial beta-defensins $D E F B 1$, $D E F B 103 A$, and DEFB104A by mucosal epithelial cells, limiting the entry of microbes through the epithelial barriers [16,17]. It contributes to virus clearance by driving the differentiation of B1a-B cells, providing for production of virüs specific $\operatorname{IgM}$ antibodies at first line of host defense [18]. IL17RA interaction with ORF8 from SARS-CoV-2 is one of the most important stages of viral infection that suppresses the immune response [4].

The discovery of effective treatments against SARS$\mathrm{CoV}-2$ requires full elucidation of the functional properties of the virus genome and proteome. Functional properties of many viral proteins, which are the target of treatment, can be achieved by in silico approaches in a shorter time and with less funds than conventional methods [19]. In this study, changes in ORF8 - ILI7RA interaction caused by mutations in SARS-CoV-2 ORF8, which play a role in the suppression of the immune response, were analyzed using in-silico approaches.

\section{Materials and Methods}

\subsection{Sequence and Mutation Data}

This study was carried out using genome data of 825 SARS CoV-2 isolates from United Kingdom (UK). Genome data of the isolates were taken from GISAID EpiCoV database [20]. Reference ORF8 accesion code is YP_009724396.1. Protein sequence information of 825 isolates were aligned with the MAFFT (v7.475) multiple sequence alignment program FFT-NS-i algorithm [21,22]. The scoring matrix BLOSUM 80 and 1 PAM was chosen for the amino acid sequences and nucleotide, respectively [23,24]. Gap opening penalty was used as 2.0. The mutated residues were analyzed with RDP4 and MegaX tools [25,26].

\subsection{Homology Model of Mutant Protein}

Three-dimensional model of mutant ORF8 protein was generated by the method of homology modeling using Swiss-Model [27]. 7JTL (RCSB protein data bank code) was selected as template. ProSA and MolProbity tools were used for structural validation and model of wild type and mutant ORF8 proteins $[28,29]$. Secondary structure components (random coils, beta strands alpha helices) of ORF8 protein were defined by using PSIPRED [30]. Superimpose and conformational analysis of wild type and mutant proteins were performed with PyMOL (ver2.4.1). Topological differences of wild type and mutant ORF8 proteins were calculated with the i-Tasser TM-Score and root mean square deviation (RMSD) algorithm [31,32].

\subsection{Docking}

The wild type and mutant ORF8 proteins were used as ligands and ILI7RA (pdb code 4HSA) was used as target for molecular docking with Haddock version 2.4. The contact points for the ILI7RA were residues number $25,26,31,87,88,89,90,91,92$, 261, 262 and 265. The contact points for the ORF8 were residues number $49,56,58,71,72,73,74$, $75,76,83,84,85$ and 95 . Number of structures for rigid body docking was set to 1000 . Number of trials for rigid body minimisation was set to 5 . Number of structures for semi-flexible refinement was set to 200. Refined with short molecular dynamics in open solvent using water. Clustering method was selected Fraction of Common Contacts (FCC). RMSD cutoff for clustering was set to $0.6 \AA$. Kyte-Doolittle hydrophobicity scale method was used for solvating. Cutoff distance (proton-acceptor) to define an hydrogen bond was set to $2.5 \AA$. Cutoff distance (carbon-carbon) to define an hydrophobic contact was set to $3.9 \AA$. Docking parameters were performed as blind docking with default values [33]. Docking results were visualized with Discovery SV (ver20.1, DDS Biovia) and PyMOL. 


\subsection{Protein Stability and Protein-Protein Affinity Analysis}

Analysis of changes in protein stability was performed using SDM2 [34], mCSM stability and DUET tools [35]. The change in ORF8 - IL17RA binding affinity before and after the mutation was evaluated using the mCSM-PPI2 tool [36].

\section{Results and Discussions}

In this study, mutation analysis of UK isolates revealed Pro30Ser, Arg52Ile, Tyr73Cys and Leu118Val mutations. The homology model of the mutant ORF8 was created with the Swiss Model. The quality scores of the mutant model were -0.94 , 0.67 and -3.83 for QMEAN, Molprobity, and ProSA, respectively. It was determined that the generated mutant ORF8 model was within the NMR quality limits (Figure 1). After docking, the TM-score (0.73) between the wild type ORF8 - IL17RA and the mutant ORF8- IL17RA complexes were observed to be topologically similar, although not high rate. The RMSD in supersposition was $0.28 \AA$. The change in the topological structure after the mutation can alter the protein-protein interaction [37].

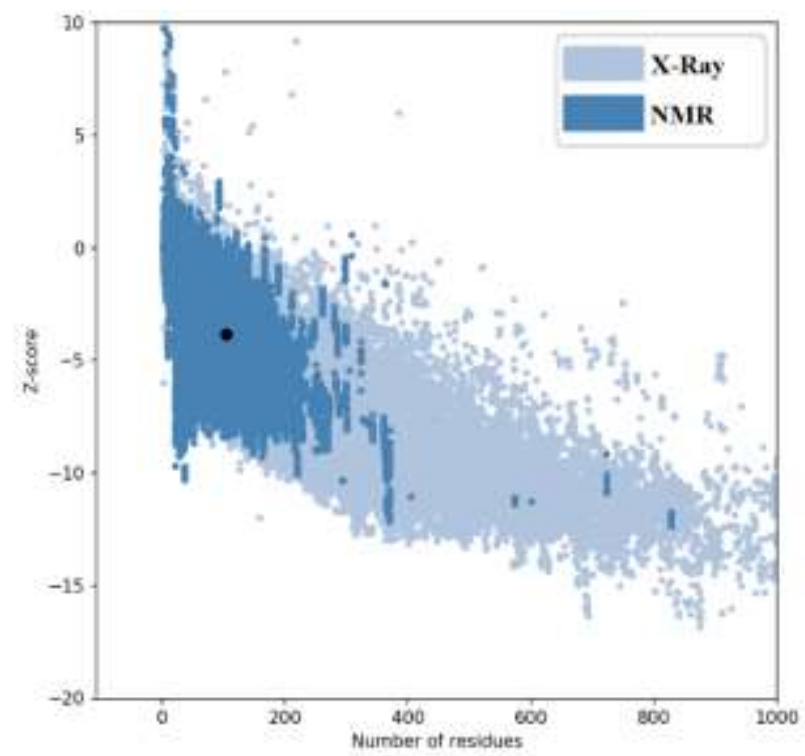

Figure 1. Quality analysis of mutant ORF8 homology model.

The docking analysis consolidated 132 structural models for the wild type ORF8 into 18 clusters, which represents $66 \%$ of the water-refined models generated. The docking analysis consolidated 129 structural models for the mutant ORF8 into 17 clusters, which represents $64 \%$ of the water-refined models generated. Docking analysis revealed the top 10 models with the lowest binding energy with IL17RA for wild type and mutant ORF8. The docking score was $-78.0 \pm 3.4 \mathrm{kcal}^{\mathrm{mol}} \mathrm{l}^{-1}$ and $-76.3 \pm 11.9 \mathrm{kcal}^{\mathrm{mol}} \mathrm{m}^{-1}$ for wild type and mutant ORF8, respectively (Table 1). Molecular docking results showed that; the ten hydrogen bonds were established between the wild type ORF8 and ILI7RA, while four hydrogen bonds were established between the mutant ORF8 and IL17RA (Table 2). The wild type ORF8 made contact with IL17RA on five locations, while between the mutant ORF8 and ILI7RA, contact was made on three locations (Figure 2). The decrease in the number of hydrogen bonds and contact points established before mutation between ORF8 from SARS-CoV-2 and ILI7RA explains the decreased affinity after the mutations.

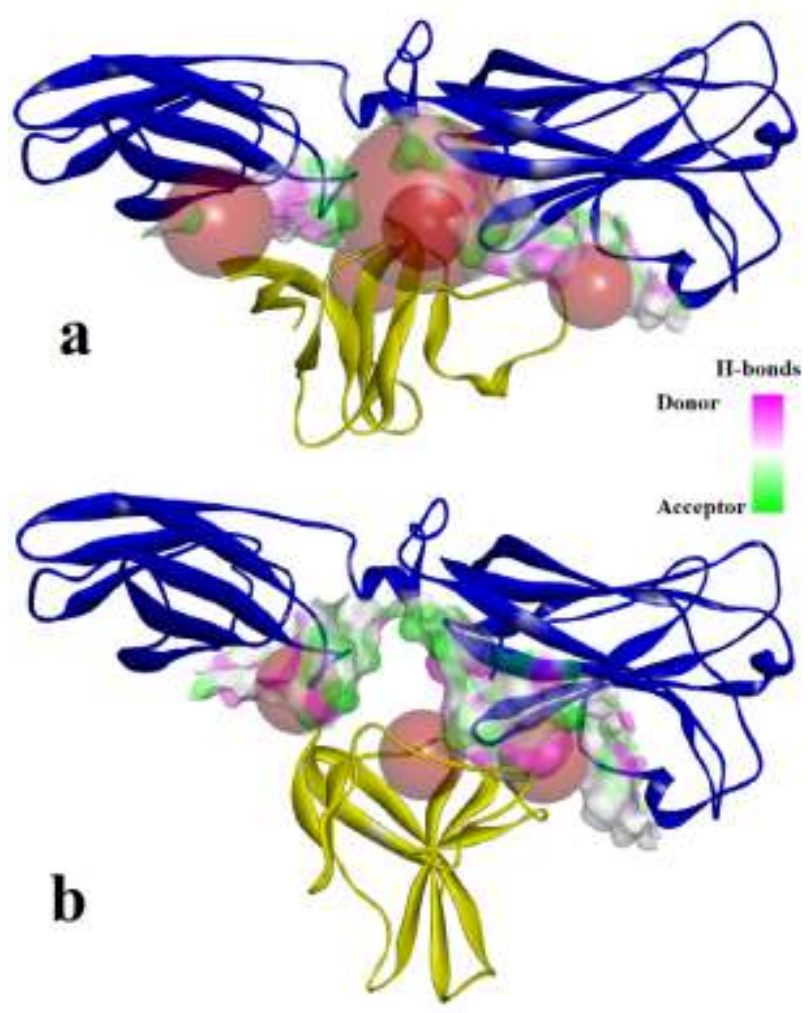

Figure 2. Bonding motif of ORF8 and IL17RA with solid ribbon presentation. a) wild type ORF8-Il17RA complex, b) mutant ORF8-IL17RA complex (red spheres represent binding sites, solid surface represents the hydrogen bond interaction surface).

Protein dynamic analysis showed that mutations in Tyr73 and Leu188 residues impair protein stability (Table 3). The $\Delta \Delta \mathrm{G}$ values were $-0.389 \mathrm{kcal}^{-\mathrm{mol}^{-1}}$ and $-0.85 \mathrm{kcal}^{\mathrm{mol}}{ }^{-1}$, respectively. However, the vibrational entropy energies between wild type and mutant showed that mutations in the Pro30, Tyr73 and Leu118 residues of the ORF8 increased protein flexibility, while the mutation at the Arg52 residue decreased flexibility (Table 3 ). The interaction data for ORF8 and IL17RA showed decreased affinity after mutation $\left(\Delta \Delta \mathrm{G}^{\text {affinity }}-0.070,-0.201,-0.924\right.$ and -1.224 for P30S, R52I, Y73C and L118V, respectively). Unlike SARS and MERS, the physical 
interaction of ORF8 from SARS-CoV-2 with ILI7RA may be associated with the high virulence effect of SARS-CoV-2 and the severe clinical course of the disease [38-41].

Although ORF8 is known to modulate the systemic IL17 signaling pathway in SARS-CoV-2 infections, its mechanism has not been fully elucidated. $I L 17$, a pleitropic cytokine, is likely to play different roles in the immune system during SARS-CoV-2 infection. This makes solving the molecular mechanism of ILI7A and SARS-CoV-2 ORF8 interaction more difficult [42]. Inactivation of ILI7RA showed that it resulted in a significant reduction in SARS-CoV-2 viral replication in ACE2 cell lines. In this study, it was shown that the region where ORF8 from SARSCoV-2 binds with the lowest binding energy on ILI7RA is the binding site of ILI7F [13]. The regulatory effect of unique signaling properties of $I L 17 R A$ on innate and adaptive immune systems is a result of its interaction with ILI7F [43,44]. Disruption of the interaction between IL17RA and $I L 17 F$ by the ORF8 linkage may be one of the main mechanisms explaining the process of suppressing the immune response. Engaging the binding site of IL17RA, a common receptor for the ILI7 family, with ORF8 may suppress the cytokine signaling process. One of regions of sequence unique to SARS-CoV-2 begins after Cys61 and extends until before the Cys83-Leu84-Pro85 conserved motif. SARS-CoV-2-specific ${ }^{73} \mathrm{YIDI}^{76}$ motif occurs at the center of this region. The YIDI motif is responsible for stabilizing an extensive non-covalent dimer interface [3]. The combination of Leu95, Ile58, Val49, and Pro56 form a hydrophobic interaction with Tyr73 of the YIDI motif. In this study, it was shown that the Tyr73Cys mutation caused an increase in flexibility and a decrease in stabilization. It appears that the occurring mutation of Tyr73 may adversely affect the stabilization of the dimer interface. This may affect the functional properties of ORF8 from SARS-CoV-2. Mutations can alter virulence characteristics of SARS-CoV-2 and affect the clinical course of the disease. The clinical effect of deletions in ORF8 may be a milder infection with less systemic release of proinflammatory cytokines and a more efficient immune response to SARS$\mathrm{CoV}-2$ [42,45].

In conclusion, it was shown in this study that 4 mutations identified in ORF8 from SARS-CoV-2 caused changes in the dynamic properties of the protein and this may result in a decrease in ILI7RA affinity. These data will contribute to elucidating the functional properties of ORF8, which is an important therapeutic target. More studies are needed to fully elucidate how ORF8 from SARS-CoV-2 suppresses the host immune system.

\section{Author Statements:}

- The authors declare that they have equal right on this paper.

- The authors declare that they have no known competing financial interests or personal relationships that could have appeared to influence the work reported in this paper

- Acknowledgement: We gratefully acknowledge the following Authors (See document S1 in Supplementary Material for authors and laboratories that provided genome data used in this study) from the Originating laboratories responsible for obtaining the specimens, as well as the Submitting laboratories where the genome data were generated and shared, on which this research is based. All submitters of data may be contacted directly via www.gisaid.org.

Table 1. Docking scores of wild and mutant ORF8 with ILI7RA

\begin{tabular}{|c|c|c|c|c|c|c|c|c|}
\hline \multirow{2}{*}{$\mathbf{1}$} & & DocSc & i-RMSD & Evdw & Eelec & Edesolv & Eair & Z-Score \\
\hline \multirow{2}{*}{$\mathbf{2}$} & $\mathbf{W}$ & $-78.0 \pm 3.4$ & $2.4 \pm 0.4$ & $-40.9 \pm 5.1$ & $-220.6 \pm 34.4$ & $-1.3 \pm 3.4$ & $83.5 \pm 25.7$ & -2.1 \\
\hline \multirow{2}{*}{$\mathbf{2}$} & $\mathbf{M}$ & $-76.3 \pm 11.9$ & $11.9 \pm 0.1$ & $-48.2 \pm 4.1$ & $-164.5 \pm 25.7$ & $-1.2 \pm 3.0$ & $60.4 \pm 40.1$ & -1.8 \\
\cline { 2 - 9 } & $\mathbf{M}$ & $-71.8 \pm 6.5$ & $14.0 \pm 0.4$ & $-35.0 \pm 7.0$ & $-255.8 \pm 26.1$ & $0.4 \pm 3.1$ & $139.1 \pm 41.0$ & -1.2 \\
\hline \multirow{2}{*}{$\mathbf{3}$} & $\mathbf{W}$ & $-66.5 \pm 8.0$ & $8.3 \pm 0.6$ & $-35.8 \pm 3.3$ & $-186.5 \pm 29.5$ & $1.5 \pm 1.7$ & $51.3 \pm 38.9$ & -0.4 \\
\cline { 2 - 9 } & $\mathbf{M}$ & $-65.8 \pm 11.8$ & $11.4 \pm 0.7$ & $-37.1 \pm 5.9$ & $-189.3 \pm 73.7$ & $1.5 \pm 2.3$ & $76.0 \pm 43.8$ & -0.4 \\
\hline \multirow{2}{*}{$\mathbf{4}$} & $\mathbf{W}$ & $-66.4 \pm 3.6$ & $6.7 \pm 1.2$ & $-47.5 \pm 10.9$ & $-160.2 \pm 43.6$ & $5.4 \pm 2.4$ & $76.9 \pm 14.0$ & -0.4 \\
\cline { 2 - 9 } & $\mathbf{M}$ & $-64.4 \pm 5.3$ & $13.6 \pm 0.3$ & $-41.1 \pm 2.8$ & $-165.3 \pm 24.1$ & $2.5 \pm 2.6$ & $72.9 \pm 31.3$ & -0.2 \\
\hline
\end{tabular}




\begin{tabular}{|c|c|c|c|c|c|c|c|c|}
\hline \multirow{2}{*}{5} & $\mathbf{W}$ & $-63.4 \pm 2.4$ & $13.1 \pm 0.1$ & $-32.1 \pm 5.9$ & $-198.1 \pm 14.5$ & $8.0 \pm 1.8$ & $3.5 \pm 3.2$ & -0.0 \\
\cline { 2 - 9 } & $\mathbf{M}$ & $-61.2 \pm 4.5$ & $10.9 \pm 0.0$ & $-31.6 \pm 9.1$ & $-253.9 \pm 23.1$ & $12.2 \pm 2.1$ & $90.1 \pm 21.0$ & 0.2 \\
\hline \multirow{2}{*}{$\mathbf{6}$} & $\mathbf{W}$ & $-60.3 \pm 8.2$ & $14.1 \pm 0.3$ & $-33.3 \pm 4.7$ & $-179.6 \pm 24.9$ & $-2.7 \pm 4.6$ & $116.7 \pm 55.7$ & 0.4 \\
\cline { 2 - 9 } & $\mathbf{M}$ & $-60.0 \pm 7.8$ & $5.1 \pm 0.4$ & $-32.1 \pm 3.2$ & $-214.9 \pm 50.4$ & $9.0 \pm 3.1$ & $61.1 \pm 28.6$ & 0.3 \\
\hline \multirow{2}{*}{$\mathbf{7}$} & $\mathbf{W}$ & $-58.8 \pm 6.3$ & $11.3 \pm 0.0$ & $-42.0 \pm 8.2$ & $-112.3 \pm 32.6$ & $0.8 \pm 2.2$ & $48.1 \pm 20.1$ & 0.6 \\
\cline { 2 - 9 } & $\mathbf{M}$ & $-59.9 \pm 5.4$ & $13.8 \pm 0.1$ & $-31.8 \pm 1.7$ & $-220.6 \pm 25.5$ & $3.4 \pm 1.3$ & $125.5 \pm 39.0$ & 0.4 \\
\hline \multirow{2}{*}{$\mathbf{8}$} & $\mathbf{W}$ & $-57.8 \pm 5.4$ & $12.5 \pm 0.7$ & $-32.8 \pm 3.8$ & $-180.0 \pm 52.8$ & $4.4 \pm 4.8$ & $66.8 \pm 60.1$ & 0.8 \\
\cline { 2 - 9 } & $\mathbf{M}$ & $-58.2 \pm 1.7$ & $8.1 \pm 0.7$ & $-41.5 \pm 4.9$ & $-120.8 \pm 11.6$ & $2.5 \pm 1.4$ & $49.9 \pm 23.9$ & 0.6 \\
\hline \multirow{2}{*}{$\mathbf{9}$} & $\mathbf{W}$ & $-56.8 \pm 5.9$ & $13.3 \pm 0.3$ & $-38.5 \pm 3.9$ & $-155.9 \pm 10.5$ & $-1.1 \pm 0.3$ & $139.7 \pm 63.1$ & 0.9 \\
\cline { 2 - 9 } & $\mathbf{M}$ & $-53.4 \pm 10.5$ & $7.4 \pm 0.5$ & $-35.3 \pm 7.1$ & $-176.7 \pm 22.2$ & $-1.7 \pm 0.6$ & $188.8 \pm 10.7$ & 1.2 \\
\hline \multirow{2}{*}{$\mathbf{1 0}$} & $\mathbf{W}$ & $-53.5 \pm 1.7$ & $10.5 \pm 0.2$ & $-39.7 \pm 3.5$ & $-164.0 \pm 42.1$ & $10.3 \pm 2.9$ & $86.8 \pm 14.9$ & 1.4 \\
\cline { 2 - 9 } & $\mathbf{M}$ & $-52.1 \pm 3.8$ & $11.2 \pm 0.2$ & $-36.2 \pm 3.8$ & $-136.4 \pm 33.7$ & $4.6 \pm 4.0$ & $67.7 \pm 32.3$ & 1.4 \\
\hline
\end{tabular}

DocSc: docking score, i-RMSD: interface RMSD (from the overall lowest-energy structure), Evdw: Van der Waals energy, Eelec: electrostatic energy, Edesolv: desolvation energy, Eair:restraints violation energy.

Table 2. Hydrogen bond interaction for ORF8-IL17RA complexes

\begin{tabular}{|c|c|c|}
\hline $\begin{array}{c}\text { Hydrogen } \\
\text { bond }\end{array}$ & IL17RA & ORF8 \\
\hline \multirow{4}{*}{ wild type } & Asn91:HN & Gln72:O \\
\cline { 2 - 3 } & Asn91:O & Gln72:HN \\
\cline { 2 - 3 } & Arg93:HH21 & Ser69:O \\
\cline { 2 - 3 } & Asp123:OD2 & Lys94:HZ3 \\
\cline { 2 - 3 } & Gln124: OE1 & Lys94:HZ1 \\
\cline { 2 - 3 } & Gln200:HE22 & Asp119:OD2 \\
\cline { 2 - 3 } & Asn261:OD1 & Glu92:HN \\
\cline { 2 - 3 } & Asp262:OD1 & Gln27:HE22 \\
\cline { 2 - 3 } & Asp262:OD2 & Gln91:HE22 \\
\hline \multirow{5}{*}{ mutant } & Asn89:O & Ile74:HN \\
\cline { 2 - 3 } & Asp121:OD2 & Lys94:HZ2 \\
\cline { 2 - 3 } & Asp121:OD2 & Lys94:HZ3 \\
\cline { 2 - 3 } & Ser257:O & His28:HE2 \\
\hline
\end{tabular}

Table 3. Effects of mutant residues on protein dynamics.

\begin{tabular}{|c|c|c|c|c|c|c|c|c|c|}
\hline \multirow[b]{2}{*}{ wild } & \multirow[b]{2}{*}{ position } & \multirow[b]{2}{*}{ mutant } & \multicolumn{5}{|c|}{$\begin{array}{c}\Delta \Delta G \\
\left(k^{-1} \text { cal.mol }\right. \\
\end{array}$} & \multirow{2}{*}{$\begin{array}{c}\Delta \Delta S \\
\left(\text { kcal.mol }^{-1} \cdot K^{-1}\right) \\
\text { ENCoM }\end{array}$} & \multirow[b]{2}{*}{ Flex. } \\
\hline & & & ENCoM & DynaMut & mCSM & SDM & DUET & & \\
\hline Pro & 30 & Ser & -0.144 & 0.146 & -1.000 & 0.810 & -0.372 & 0.180 & Inc \\
\hline Arg & 52 & Ile & 0.124 & 0.436 & 0.206 & 0.910 & 0.414 & -0.155 & Dec \\
\hline Tyr & 73 & Cys & -0.525 & -0.389 & -0.526 & -0.040 & -0.290 & 0.656 & Inc \\
\hline Leu & 118 & Val & -0.248 & -0.850 & -1.593 & -1.580 & -1.712 & 0.310 & Inc \\
\hline
\end{tabular}

Flex.: Flexibility 


\section{References}

[1] Worldometer, "Coronavirus Cases" Worldometer (2020) $1-22$. https://doi.org/10.1101/2020.01.23.20018549V2.

[2] P. Zhou, X. Lou Yang, X.G. Wang, B. Hu, L. Zhang, W. Zhang, H.R. Si, Y. Zhu, B. Li, C.L. Huang, H.D. Chen, J. Chen, Y. Luo, H. Guo, R. Di Jiang, M.Q. Liu, Y. Chen, X.R. Shen, X. Wang, X.S. Zheng, K. Zhao, Q.J. Chen, F. Deng, L.L. Liu, B. Yan, F.X. Zhan, Y.Y. Wang, G.F. Xiao, Z.L. Shi, "A pneumonia outbreak associated with a new coronavirus of probable bat origin" Nature 579 (2020) 270-273. https://doi.org/10.1038/s41586020-2012-7.

[3] T.G. Flower, C.Z. Buffalo, R.M. Hooy, M. Allaire, X. Ren, J.H. Hurley, "Structure of SARS-CoV-2 ORF8, a rapidly evolving coronavirus protein implicated in immune evasion" Proceedings of the National Academy of Sciences 118 (2020) 1-6. https://doi.org/10.1101/2020.08.27.270637.

[4] D.E. Gordon, D.E. Gordon, J. Hiatt, M. Bouhaddou, V. V Rezelj, S. Ulferts, "Comparative hostcoronavirus protein interaction networks reveal panviral disease mechanisms" Science 9403 (2020) 138.

[5] A. Fontanet, B. Autran, B. Lina, M.P. Kieny, S.S.A. Karim, D. Sridhar, "SARS-CoV-2 variants and ending the COVID-19 pandemic" The Lancet 397 (2021) 952-954. https://doi.org/10.1016/S01406736(21)00370-6.

[6] M. Miao, E. De Clercq, G. Li, "Genetic Diversity of SARS-CoV-2 over a One-Year Period of the COVID-19 Pandemic: A Global Perspective" Biomedicines $\quad 9 \quad 412$. https://doi.org/10.3390/biomedicines9040412.

[7] F. Wu, S. Zhao, B. Yu, Y.M. Chen, W. Wang, Z.G. Song, Y. Hu, Z.W. Tao, J.H. Tian, Y.Y. Pei, M.L. Yuan, Y.L. Zhang, F.H. Dai, Y. Liu, Q.M. Wang, J.J. Zheng, L. Xu, E.C. Holmes, Y.Z. Zhang, "A new coronavirus associated with human respiratory disease in China" Nature 579 (2020) 265-269. https://doi.org/10.1038/s41586-020-2008-3.

[8] F. Pereira, "Evolutionary dynamics of the SARSCoV-2 ORF8 accessory gene" Infection, Genetics and Evolution $85 \quad$ (2020) 1-10. https://doi.org/10.1016/j.meegid.2020.104525.

[9] C. Baruah, P. Devi, D.K. Sharma, "Sequence analysis and structure prediction of SARS-CoV-2 accessory proteins 9b and ORF14: Evolutionary analysis indicates close relatedness to bat coronavirus" BioMed Research International 2020 (2020) 1-13. https://doi.org/10.1155/2020/7234961.

[10] Y. Zhang, J. Zhang, Y. Chen, B. Luo, Y. Yuan, F. Huang, T. Yang, F. Yu, J. Liu, B. Liu, Z. Song, J. Chen, T. Pan, X. Zhang, Y. Li, R. Li, W. Huang, F. Xiao, H. Zhang, "The ORF8 protein of SARS-CoV2 mediates immune evasion through potently downregulating MHC-I" BioRxiv (2020) 1-41. https://doi.org/10.1101/2020.05.24.111823.

[11] et al. Gordon DE, Jang GM, Bouhaddou M, "A SARS-CoV-2 protein interaction map reveals targets for drug repurposing" Nature 583 (2020) 1-13.

[12] J.Y. Li, C.H. Liao, Q. Wang, Y.J. Tan, R. Luo, Y. Qiu, X.Y. Ge, "The ORF6, ORF8 and nucleocapsid proteins of SARS-CoV-2 inhibit type I interferon signaling pathway" Virus Research 286 (2020) 198074. https://doi.org/10.1016/j.virusres.2020.198074.

[13] L.K. Ely, S. Fischer, K.C. Garcia, "Structural basis of receptor sharing by interleukin 17 cytokines" Nature Immunology 10 (2009) 1245-1251. https://doi.org/10.1038/ni.1813.

[14] D. Toy, D. Kugler, M. Wolfson, T. Vanden Bos, J. Gurgel, J. Derry, J. Tocker, J. Peschon, "Cutting Edge: Interleukin 17 Signals through a Heteromeric Receptor Complex" The Journal of Immunology 177 (2006) https://doi.org/10.4049/jimmunol.177.1.36.

[15] M. Kurte, P. Luz-Crawford, A.M. Vega-Letter, R.A. Contreras, G. Tejedor, R. Elizondo-Vega, L. Martinez-Viola, C. Fernández-O'Ryan, F.E. Figueroa, C. Jorgensen, F. Djouad, F. Carrión, "IL17/IL17RA as a novel signaling axis driving mesenchymal stem cell therapeutic function in experimental autoimmune encephalomyelitis" Frontiers in Immunology 9 (2018) 802. https://doi.org/10.3389/fimmu.2018.00802.

[16] V. Ramirez-Carrozzi, A. Sambandam, E. Luis, Z. Lin, S. Jeet, J. Lesch, J. Hackney, J. Kim, M. Zhou, J. Lai, Z. Modrusan, T. Sai, W. Lee, M. Xu, P. Caplazi, L. Diehl, J. De Voss, M. Balazs, L. Gonzalez, H. Singh, W. Ouyang, R. Pappu, "IL-17C regulates the innate immune function of epithelial cells in an autocrine manner" Nature Immunology 12 (2011) 1159-1166. https://doi.org/10.1038/ni.2156.

[17] B. Neupane, D. Acharya, F. Nazneen, G. GonzalezFernandez, A.S. Flynt, F. Bai, "Interleukin-17A Facilitates Chikungunya Virus Infection by Inhibiting IFN- $\alpha 2$ Expression" Frontiers in Immunology $\quad 11 \quad$ (2020) 2955. https://doi.org/10.3389/fimmu.2020.588382.

[18] W.T. Ma, X.T. Yao, Q. Peng, D.K. Chen, "The protective and pathogenic roles of IL-17 in viral infections: Friend or foe?" Open Biology 9 (2019) 190109. https://doi.org/10.1098/rsob.190109.

[19] V.K. Bhardwaj, R. Singh, P. Das, R. Purohit, "Evaluation of acridinedione analogs as potential SARS-CoV-2 main protease inhibitors and their comparison with repurposed anti-viral drugs" Computers in Biology and Medicine 128 (2021) 113.

https://doi.org/10.1016/j.compbiomed.2020.104117

[20] GISAID, "GISAID - Next hCoV-19 App" Genomic Epidemiology of HCoV-19 (2020). https://www.gisaid.org (accessed April 1, 2021).

[21] K. Katoh, "MAFFT: a novel method for rapid multiple sequence alignment based on fast Fourier transform" Nucleic Acids Research 30 (2002) 30593066. https://doi.org/10.1093/nar/gkf436. 
[22] K. Katoh, J. Rozewicki, K.D. Yamada, "MAFFT online service: Multiple sequence alignment, interactive sequence choice and visualization" Briefings in Bioinformatics 20 (2018) 1160-1166. https://doi.org/10.1093/bib/bbx108.

[23] D.W. Mount, "Using BLOSUM in sequence alignments" Cold Spring Harbor Protocols 3 (2008) pdb-top39. https://doi.org/10.1101/pdb.top39.

[24] D.W. Mount, "Using PAM matrices in sequence alignments" Cold Spring Harbor Protocols 3 (2008) 1-9. https://doi.org/10.1101/pdb.top38.

[25] D.P. Martin, B. Murrell, M. Golden, A. Khoosal, B. Muhire, "RDP4: Detection and analysis of recombination patterns in virus genomes" Virus Evolution 1

(2015). https://doi.org/10.1093/ve/vev003.

[26] S. Kumar, G. Stecher, M. Li, C. Knyaz, K. Tamura, "MEGA X: Molecular evolutionary genetics analysis across computing platforms" Molecular Biology and Evolution 35 (2018) 1547-1549. https://doi.org/10.1093/molbev/msy096.

[27] A. Waterhouse, M. Bertoni, S. Bienert, G. Studer, G. Tauriello, R. Gumienny, F.T. Heer, T.A.P. De Beer, C. Rempfer, L. Bordoli, R. Lepore, T. Schwede, "Swiss-Model: Homology modelling of protein structures and complexes" Nucleic Acids Research $46 \quad$ (2018) W296-W303. https://doi.org/10.1093/nar/gky427.

[28] M. Wiederstein, M.J. Sippl, "ProSA-web: Interactive web service for the recognition of errors in three-dimensional structures of proteins" Nucleic Acids Research (2007). https://doi.org/10.1093/nar/gkm290.

[29] V.B. Chen, W.B. Arendall, J.J. Headd, D.A. Keedy, R.M. Immormino, G.J. Kapral, L.W. Murray, J.S. Richardson, D.C. Richardson, "MolProbity: Allatom structure validation for macromolecular crystallography" Acta Crystallographica Section D: Biological Crystallography 66 (2010) 12-21. https://doi.org/10.1107/S0907444909042073.

[30] D.W.A. Buchan, F. Minneci, T.C.O. Nugent, K. Bryson, D.T. Jones, "Scalable web services for the PSIPRED Protein Analysis Workbench." Nucleic Acids Research $41 \quad$ (2013) W349-W357. https://doi.org/10.1093/nar/gkt381.

[31] Y. Zhang, J. Skolnick, "Scoring function for automated assessment of protein structure template quality" Proteins: Structure, Function and Genetics 57 (2004) 702-710. https://doi.org/10.1002/prot.20264.

[32] J. Xu, Y. Zhang, "How significant is a protein structure similarity with TM-score $=0.5$ ?" Bioinformatics $26 \quad$ (2010) 889-895. https://doi.org/10.1093/bioinformatics/btq066.

[33] G.C.P. Van Zundert, J.P.G.L.M. Rodrigues, M. Trellet, C. Schmitz, P.L. Kastritis, E. Karaca, A.S.J. Melquiond, M. Van Dijk, S.J. De Vries, A.M.J.J. Bonvin, "The HADDOCK2.2 Web Server: UserFriendly Integrative Modeling of Biomolecular Complexes" Journal of Molecular Biology 428 (2016) $720-725$. https://doi.org/10.1016/j.jmb.2015.09.014.

[34] A.P. Pandurangan, B. Ochoa-Montaño, D.B.
Ascher, T.L. Blundell, "SDM: A server for predicting effects of mutations on protein stability" Nucleic Acids Research 45 (2017) W229-W235. https://doi.org/10.1093/nar/gkx439.

[35] D.E. V Pires, D.B. Ascher, T.L. Blundell, "DUET: a server for predicting effects of mutations on protein stability using an integrated computational approach" Nucleic Acids Research 42 (2014) W314-W319.

[36] C.H.M. Rodrigues, Y. Myung, D.E.V. Pires, D.B. Ascher, "MCSM-PPI2: predicting the effects of mutations on protein-protein interactions" Nucleic Acids Research 47 (2019) W338-W344. https://doi.org/10.1093/nar/gkz383.

[37] D. Davis, Ö.N. Yaverołlu, N. Malod-Dognin, A. Stojmirovic, N. Pržulj, "Topology-function conservation in protein-protein interaction networks" Bioinformatics 31 (2015) 1632-1639. https://doi.org/10.1093/bioinformatics/btv026.

[38] Y. Liu, C. Zhang, F. Huang, Y. Yang, F. Wang, J. Yuan, Z. Zhang, Y. Qin, X. Li, D. Zhao, S. Li, S. Tan, Z. Wang, J. Li, C. Shen, J. Li, L. Peng, W. Wu, M. Cao, L. Xing, Z. Xu, L. Chen, C. Zhou, W.J. Liu, L. Liu, C. Jiang, "Elevated plasma levels of selective cytokines in COVID-19 patients reflect viral load and lung injury" National Science Review 7 (2020) 1003-1011. https://doi.org/10.1093/nsr/nwaa037.

[39] C. Huang, Y. Wang, X. Li, L. Ren, J. Zhao, Y. Hu, L. Zhang, G. Fan, J. Xu, X. Gu, Z. Cheng, T. Yu, J. Xia, Y. Wei, W. Wu, X. Xie, W. Yin, H. Li, M. Liu, Y. Xiao, H. Gao, L. Guo, J. Xie, G. Wang, R. Jiang, Z. Gao, Q. Jin, J. Wang, B. Cao, "Clinical features of patients infected with 2019 novel coronavirus in Wuhan, China" The Lancet 395 (2020) 497-506. https://doi.org/10.1016/S0140-6736(20)30183-5.

[40] C. Qin, L. Zhou, Z. Hu, S. Zhang, S. Yang, Y. Tao, C. Xie, K. Ma, K. Shang, W. Wang, D.S. Tian, "Dysregulation of immune response in patients with coronavirus 2019 (COVID-19) in Wuhan, China" Clinical Infectious Diseases 71 (2020) 762-768. https://doi.org/10.1093/cid/ciaa248.

[41] G. Chen, D. Wu, W. Guo, Y. Cao, D. Huang, H. Wang, T. Wang, X. Zhang, H. Chen, H. Yu, X. Zhang, M. Zhang, S. Wu, J. Song, T. Chen, M. Han, S. Li, X. Luo, J. Zhao, Q. Ning, "Clinical and immunological features of severe and moderate coronavirus disease 2019" Journal of Clinical Investigation $\quad 130 \quad$ (2020) 2620-2629. https://doi.org/10.1172/JCI137244.

[42] B.E. Young, S.W. Fong, Y.H. Chan, T.M. Mak, L.W. Ang, D.E. Anderson, C.Y.P. Lee, S.N. Amrun, B. Lee, Y.S. Goh, Y.C.F. Su, W.E. Wei, S. Kalimuddin, L.Y.A. Chai, S. Pada, S.Y. Tan, L. Sun, P. Parthasarathy, Y.Y.C. Chen, T. Barkham, R.T.P. Lin, S. Maurer-Stroh, Y.S. Leo, L.F. Wang, L. Renia, V.J. Lee, G.J.D. Smith, D.C. Lye, L.F.P. Ng, "Effects of a major deletion in the SARS-CoV-2 genome on the severity of infection and the inflammatory response: an observational cohort study" The Lancet 396 (2020) 603-611. https://doi.org/10.1016/S0140-6736(20)31757-8.

[43] A. Goepfert, S. Lehmann, J. Blank, F. Kolbinger, J.M. Rondeau, "Structural Analysis Reveals that the 
Cytokine IL-17F Forms a Homodimeric Complex with Receptor IL-17RC to Drive IL17RAIndependent Signaling" Immunity 52 (2020) 499512.e5.

https://doi.org/10.1016/j.immuni.2020.02.004.

[44] M. Veldhoen, "Interleukin 17 is a chief orchestrator of immunity" Nature Immunology 18 (2017) 612-
621. https://doi.org/10.1038/ni.3742.

[45] L. Zinzula, "Lost in deletion: The enigmatic ORF8 protein of SARS-CoV-2" Biochemical and Biophysical Research Communications 538 (2021) 116-124.

https://doi.org/10.1016/j.bbrc.2020.10.045. 